\title{
中国心理学前沿
}

\section{具身性情绪：面部表情和身体姿 势对情绪感知的影响}

\author{
许洪梅 \\ 广州大学教育学院，广州，510006 \\ 邮箱: 1130309607@qq.com
}

摘 要: 近二十年来具身认知的研究已经从思辨走向实证研究。对于具身情绪 的探讨也有了许多成果。实验室里的研究到实际的应用方面的转变还有待发展。 本文综述了具身性情绪领域的实证研究，包括面部表情和身体姿势的操纵对情 绪的影响, 姿势的改变如何调节情绪, 以及研究在情绪障碍中的临床应用。 关键词：具身认知；具身性情绪；面部表情；身体姿势

\section{Embodiment emotion: The effect of facial expression and body posture on the perception of emotion}

\section{$\mathrm{Xu}$ Hongmei}

School of Education, Guangzhou University, Guangzhou 510006

Abstract: In recent 20 years, the study of embodiment cognition has gone from 
speculation to empirical research. There are also many achievements in the discussion of embodiment emotion. The transformation from laboratory research to practical application needs to be developed. This paper reviews the empirical research in the field of embodiment emotion, including the influence of facial expression and manipulation of body posture on emotion, how the change of posture adjusts emotion, and the application of this study in emotional disorder.

Key words: Embodiment cognition; Embodiment emotion; Facial expression; Body posture

Received: 2019-06-23; Accepted: 2019-07-24; Published: 2019-07-30

Copyright (C) 2019 by author(s) and SciScan Publishing Limited

This work is licensed under a Creative Commons Attribution 4.0 International License. https://creativecommons.org/licenses/by/4.0/

\section{1 引言}

具身的思想在哲学领域的起源有一个长久的过去。从胡塞尔的现象学, 到 海德格尔的 “存在” 概念强调人与世界的一体，再到梅洛 - 庞蒂提出 “具身的 主题性” 概念, 认为知觉的主题是身体，而身体嵌人世界中。这些哲学家们都 强调身体在整体的 “我” 中所扮演的重要角色。具身认知在心理学的研究源于 20 世纪 80 年代。彼时认知心理学遭遇困境，心理学家们开始意识到人类的大脑 之外, 还有身体这个重要资源参与到个体的认知中, 开始了具身认知的研究思潮。 人类到底是如何知觉物体的? 认知仅仅是大脑的活动吗? 从古希腊时期柏拉图 伊始，人们就已经有了贬低身体的意识，认为灵魂才是至高无上的。而到了中 世纪，笛卡尔的思想实验得出我思故我在后，更为二元论思想提供了理论支撑。 如果灵魂和肉体是两个实体，他们可以互为独立，那么个体是如何成为一个完 
整的人? 身体和思想之间是如何联系以使得个体可以完成日常活动，甚至可以 感受欢乐和痛苦?

\section{2 具身情绪理论}

Darwin 最先提出外在的身体姿势和面部表情与情绪体验紧密的联系在一起 的观点，他认为情绪性的表达和神经系统的反应具有双向的作用 [1]。换言之， 在一个被操纵的身体姿态中，不管有没有情绪性事件的刺激，都可能提供外周 反馈，影响与情绪有关的生理过程。到了心理学家 James 这里，他提出人们是因 为笑才会开心, 因为哭泣才伤心 [2]。如果没有身体上的感觉，一种情绪不过 是一种冷酷的理性判断, 而这种判断不可能被贴上情感的标签。尽管 Cannon 对 此作出了强有力的批评 [3], 使得 James 的情绪理论在心理学界一直没有被广 泛接受, 但 Laird 的研究给出了足够的证据证明 James 的情绪理论是正确的 [4]。 人们感受到的情绪, 是对身体反应的感知, 身体反应先于情感体验。如果个体 不是因为伤心才哭泣, 那是为什么会哭泣呢? Laird 进一步说明, 情感行为和身 体变化是一个进化的、自动的系统，它们提供了产生情绪的线索 [4]。当使得 个体难过的事件发生时，身体开始有了反应。个体可能会皱眉，内脏开始产生 反应, 到达一定程度就会流泪, 接着感到了自己的悲伤。这同具身认知理论的 情绪观是一致的。该理论认为，对情绪的感知和思考涉及到自我内部相关情绪 的知觉, 躯体, 内脏和运动的再体验 $[5]$ 。Gallese 等人进行了一项 fMRI 研究, 参与者吸人产生强烈厌恶感的气味和观看显示厌恶情绪面部表情的视频片段, 激活了相同的部位 [6]。也就是说被试在自己感到厌恶和观看他人的感到厌恶 激活了同样的脑区, 这和具身情绪中的模拟理论是一致的。本文综合讨论心理 学实验室里面部表情和身体姿势的操纵实验, 对具身情绪理论做进一步论证。

\section{3 实验研究}

\section{1 面部表情的实验研究}

Laird 认同 James 的情绪理论 [7]，他是首先对这一理论进行实证研究的实 
验者之一。在实验中，告知被试实验目的是考察知觉过程中各种条件下的面部 肌肉活动。在实验结束时收集参与者的回答显示，绝大部分参与者没有猜测到 实验是关于情绪表达的。主试向参与者提供指令移动脸部肌肉，在参与者没有 意识的情况下创造出情绪的面部表情。第一个实验中, 给被试展示 $3 \mathrm{k}$ 党成员的 图片或者微笑的孩子的图片，诱导愤怒和快乐的情绪。结果显示，与看微笑的 孩子的图片相比，看 $3 \mathrm{k}$ 党成员的图片的被试自我报告中攻击性更高, 看微笑孩 子的照片被试报告幸福感更高。在第二个实验中，参与者保持微笑会比皱眉对 卡通图片幽默程度评分更高。一些研究者质疑 Laird 的实验结果，认为被试的要 求特征没有得到排除。是否来访者意识到自己被要求呈现一个快乐的或者悲伤 的表情呢? 为了解决上述疑问, Strack 展开另一项操纵表情的实验研究 [8]。 他让被试嘴里咬着一支笔, 当参与者用牙齿咬着笔时, 就呈现了一个微笑的表情。 当参与者用凸出的嘴唇固定笔时，微笑就被抑制了。这样就可以分散对表情的 注意力, 排除对实验目的的猜测。结果显示，用牙齿咬着笔时，被试觉得卡通 片更好笑。Strack 的研究表明, 面部表情影响情绪的反应, 实验结果不能被要求 特征解释。Soussignan 的研究也得到了相似的结果 [9]。总而言之, 微笑使得 个体更积极，波眉使得个体更消极。两实验的面部表情操纵图如图 1 所示 [5]。

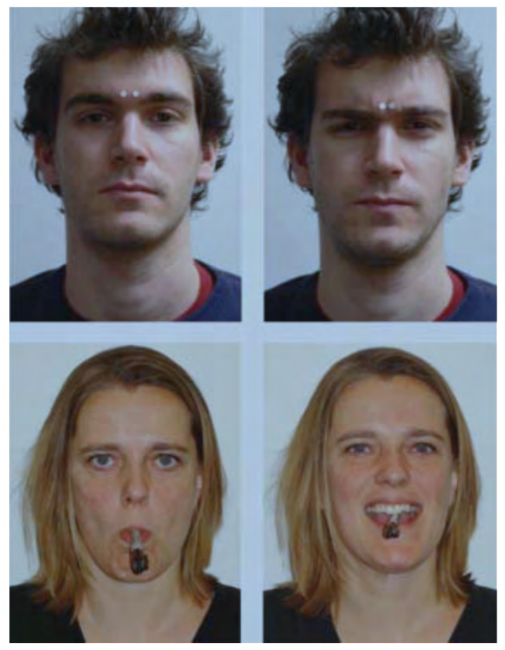

图 1 面部肌肉操纵

Figure 1 Manipulation of facial muscles 
另一类研究是关于抑制表情展现的研究，研究者发现抑制表情的个体会减 弱相关的情感体验。Duclos 等人发现，参与者抑制面部表情的表达时会降低消 极的情感 $[10]$ 。Bush 等人的研究结果显示, 当参与者抑制面部表情的表达时, 会降低积极的情感 [11]。Mccanne等人则发现, 积极和消极的情感都会减少 [12］。 这些关于面部表情的研究证实了身体状态可以影响主观的情绪体验，而不是由 于认知因素使情绪得以产生。在 Levenson 等人的研究中, 被试的面部表情被实 验者由指导语操纵，然后开始一系列的生理指标的测量，比如心率，皮肤电导 率，手指温度和前臂肌肉张力 [13]。参与者的自我报告的情绪也被记录下来。 结果显示，愤怒，恐惧和戻恶的表达在收集的自主神经测量中具有独特的生理 反应模式。这说明，面部表情不仅影响了个体的情绪表达，也影响了内部的生 理活动。

\section{2 身体姿势操纵的研究}

Izard 和 James 认为，一个人身体的姿势可能会对他的情绪体验和内心状态 产生反馈效应［2］［14］。1982 年 Riskind 等人开启实验室操纵身体姿势（直 立坐姿或者弯腰坐姿）研究的先河 [15]，他进行了四项实验研究，以检验身 体姿势的变化是否具有调节或反馈作用，影响动机和情绪。姿势操作图片如图 2 所示 $[15]$ 。第一项研究发现弯腰坐姿的被试在其后的挫折任务中坚持时间更短, 似乎更容易产生无助感。第二项研究改变了被试，得到了相同的结果。所有实 验都确保实验者和被试都不知道实验的具体操纵目的。令人惊讶的是, 在两项 实验里，被试的情绪自我报告都没有差异。第三项研究证实，身体姿势是观察 者对知觉他人情绪的重要线索。第四项研究进一步探讨了姿势在情绪报告中的 作用。对于为什么没有得到情绪的差异问题，研究者根据前人的相关研究认为， 被试虽然在自我报告中没有表现出情绪差异，但在随后的行为指标上表现出了 差异，时间短暂情绪信息未能被感知 [16]。 


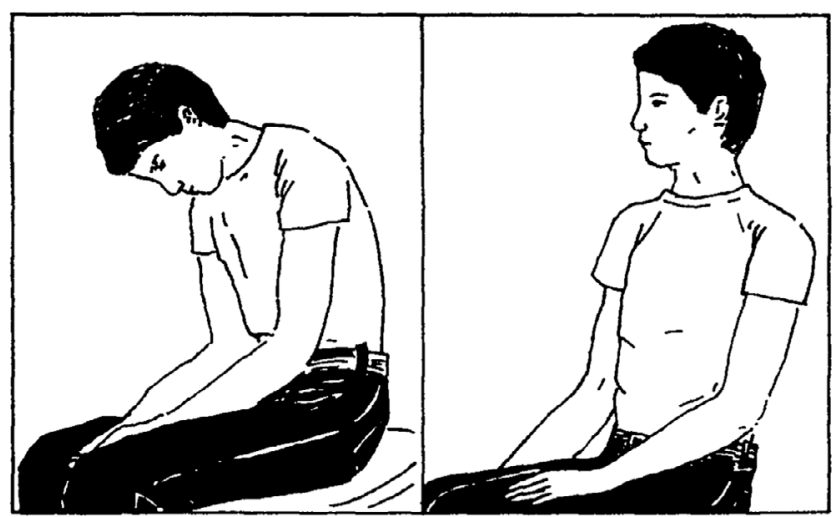

图 2 弯腰和直立姿势

Figure 2 Posture of slumped and upright

1984 年 Riskind 再次进行直立和弯腰坐姿的实验研究 $[17]$ ，这次他们不仅 改变了单一的情绪测量手段，而且还考虑了两种坐姿的舒适度，并进行排除。 他们发现人体的这两种姿势不仅是精神状态的被动指标，而且可以影响一个人 的心理状态和行为。在研究中, 当坐姿和情绪不匹配时 (比如弯腰坐姿和成功 的反馈）会具有较少动机以及控制的感觉。Michalak 等人探讨了不同坐姿状态, 抑郁症患者对正负性回忆的偏向性 [18］。他们发现直立坐姿下，对正负性词 汇的回忆不带有偏向，而弯腰状态下病人表现出更多的负性词的回忆。研究表 明了人体姿态的改变可以影响抑有症患者的认知偏差。Veenstra 则进一步研究了 两种姿态中对消极情绪恢复的影响 [19］。研究发现在弯腰姿态下，情绪恢复 较少。他们的第二个实验证明了无论参与者是否进行认知重新评估，姿势都有 影响情绪恢复的效果。因此，姿势对情绪的影响似乎是通过与认知重新评估不 同的机制来实现的。姿势可以被看作是一种替代的渠道，人们可以通过它来调 节自己的情绪状态，这是一种与认知形式的情绪调节平行运作的渠道。Nair 等 人研究了直立和弯腰坐姿对心理应激任务的情绪和心血管反应是否有影响 [20 ]。 研究过程中, 参与者的背部被用物理治疗胶带绑住, 以保持姿势。参与者完成 了一项阅读任务, 社会压力演讲任务, 然后进行情绪与自尊和感觉到的威胁的 评估。同时对血压和心率进行连续测量。结果发现与弯腰坐姿的参与者相比, 直立坐姿的被试表现出更高的自尊、更多的兴奋、更积极的情绪和更低的恐惧。 
直立姿势的受试者在应激期间和应激后的血压较高。他们的研究结果表明，面 对压力时采用直立坐姿能保持自尊, 减少消极情绪, 并能提高积极情绪。此外, 直立坐姿能提高说话的几率，减少自我专注。所以坐姿可能是一种简单的行为 策略, 可以帮助培养抗压能力。这一研究与肌肉和自主神经状态影响情绪反应 的具身化认知理论同样是一致的。

\section{4 具身情绪调节的研究}

Shafir 等人检验了对运动的蓄意控制可以调节情绪这个可能性 [21］。他们 假设参与某种情绪相关的运动会增强这种情绪的相应的效价。由于想象的运动 表象和观察运动被认为激活了在运动过程中参与的同一个镜像神经元，他们也 可能激活相同的情感处理回路，从而产生相似的情感体验。于是他们测量了运 动执行，运动意向和整体情绪的动态表达（快乐，悲伤，恐惧）对情绪状态的 影响。三项任务都增强了相应的情感状态，表明了他们调节情绪的潜力。即运 动执行、全身情绪运动的观察和表象都可以增强相应的情感状态，从而有助于 调节自己的情绪和识别他人的情绪。已经提到过的 Veenstra 等人的研究也表明, 改变情绪表达（即一种姿势）可以是调节情绪的一种有效方式 [22］。特别是 当研究中的参与者不知道他们的姿势与其情绪之间的联系时。所以，不同的坐 姿间接地调节了人们的情绪状态。Osypiuk 等则论证了动、静态身体姿势是太极 拳、气功等心身练习的一个决定性特征 [23]。越来越多的证据支持这一假设, 即太极和气功的练习有利于心理健康，包括抑郁和焦虑的管理和预防。虽然各 种因素被确定为这种健康益处的潜在中介因素，但身体姿势的表现尤为突出。 研究者推测, 站立或移动时的身体姿势是太极和气功对心理健康影响的关键治 疗因素。在文章中, 作者总结了现有的实验和观察证据, 表明身体姿势与心理 状态之间存在双向关系。另一项对 68 名女性成年的研究表明 [24]，在进行镜 像暴露 (饮食失调症治疗的主要组成部分) 前, 采取不同的姿势对身体相关情 绪和认知有影响。采取扩展姿势的女性, 在进行镜像暴露后表现出较高的积极 情绪, 提高了身体形象满意度和对自己身体的评价。这些实证研究都表明了身 体姿势在调节情绪中的显著作用，具身情绪的这种调节作用可以为情绪领域的 
研究提供不同视角。

\section{5 研究应用}

通过上述的实证研究，可以看到身体姿势和面部表情与情绪体验之间具有 双向作用。这给临床运用以启示：许多心理疾病的治疗也许并非仅仅依靠心理 咨询理论的指导，由于身心一体特征，改变身体的运动方式也可能会促进心理 的改善，治愈包括抑郁症，失眠，孤独症等在内的多种疾病 [25]。Wilkes 等 人对 61 名轻中度抑郁症患者的研究表明，直立坐姿可以改善抑郁症患者的情绪 和疲劳程度，较少自我专注的程度 [26]。

身体往往比精神状态或情绪更容易控制，通过学习可以意识到社会互动的 非语言的、具体的组成部分，抑郁的个体可以通过干预情绪传染的过程来保持 他们社会互动的质量。有意识地努力保持开放、开阔的身体姿势，并以一种充 满活力的方式微笑和行动是有用的。尽管这些努力可能与内心的情绪状态不一 致，但是对他人呈现出积极情绪，自己会产生积极的情绪，从而对社会互动的 质量产生巨大的影响。虽然这里的焦点是社会互动，但已经被讨论过的姿势和 面部表情对个体的情绪也有重要的自下而上的影响。此外，经常使用的肌肉群 倾向于加强，而不使用的则倾向于萎缩 [27］。因此，随着时间的推移，悲伤 的重复身体表达可能会变成无意识的，导致情绪下降和这种情绪持续的趋势， 以及更难像前面所描述的那样有意识地表达积极的影响。值得注意的一点是, 有意识地用积极的表达代替悲伤的表达可能是最有价值的。虽然负面情绪的面 部和身体过度表达可能导致消极情绪的躯体启动，但也有必要在此与表达真实 情绪的需要之间取得平衡。Dijkstra、Kaschak 和 Zwaan 研究表明，当姿势与记忆 相关的情绪相一致时，记忆更容易恢复 [28]。因此，姿势加上步态和面部表情， 这些积极情绪的特征可能会导致积极记忆的优先回忆，改善抑郁回忆偏见 [29］。

综上所述，具身情绪理论的应用有助于改善情绪障碍患者的社会功能，但 在涉及社会功能的疾病中的直接应用仍处于初级阶段。基础研究的积累表明, 这一领域将是一个很有前途的领域。对社会认知的更广泛、更具体、更深人的 视角将有助于解决重要的临床问题。这一领域的未来研究最好通过结合社会认 
知研究方向、临床和其他应用领域的专家共同努力加以解决。文章仅讨论了身 体在情绪中所起到的重要作用, 但具身认知同样强调社会环境的作用。社会环 境和身体，综合起来传达给个体的信息，形成了情绪的感知，个体因此感受到 情绪。总之, 具身性情绪理论给了研究者不同视角更好地理解人类的情绪, 这 一领域的研究与应用还有待继续发展。

\section{参考文献}

[1] Darwin C. Expression of the emotions in man and animals [ M ] //In The history of psychology: Fundamental questions. New York: Oxford University Press, 2003.

[2] James W. What is an emotion? [ J ] . Mind, 1884, 9 ( 34 ) : 188-205. https://doi.org/10.1093/mind/os-IX.34.188

[3] Cannon W B. The James-Lange theory of emotions: A critical examination and an alternative theory $[\mathrm{J}]$. The American journal of psychology, 1927, 39( 1/4 ): 106-124. https://doi.org/10.2307/1415404

[4] Laird J D, Lacasse K. Bodily influences on emotional feelings: accumulating evidence and extensions of William James's theory of emotion [ $\mathrm{J}]$. Emotion Review, 2014, 6 ( 1 ) : 27-34. https://doi.org/10.1177/1754073913494899

[5] Niedenthal P M. Embodying emotion [ J ] . Science, 2007, 316 ( 5827) : 1002-1005. https://doi.org/10.1126/science.1136930

[6] Gallese V, Royet J P, Plailly J, et al. Both of us disgusted in my insula: the common neural basis of seeing and feeling disgust $[\mathrm{J}]$. Neuron, 2003, 40 ( 3 ): 655-664. https://doi.org/10.1016/S0896-6273(03)00679-2

[7] Laird J D. Self-attribution of emotion: the effects of expressive behavior on the quality of emotional experience $[\mathrm{J}]$. Journal of Personality \& Social Psychology, 1974, 29 ( 4 ) : 475-86. https://doi.org/10.1037/h0036125

[ 8 ] Strack F, Martin L L, Stepper S. Inhibiting and facilitating conditions of the human smile: a nonobtrusive test of the facial feedback hypothesis $[\mathrm{J}]$. 
Journal of Personality and Social Psychology, 1988, 54 ( 5 ) : 768-777.

https://doi.org/10.1037/0022-3514.54.5.768

[9] Soussignan R. Duchenne smile, emotional experience, and autonomic reactivity: a test of the facial feedback hypothesis $[\mathrm{J}]$. Emotion, 2002, 2( 1 ): 52-74. https://doi.org/10.1037//1528-3542.2.1.52

[ 10 ] Duclos S E, Laird J D. The deliberate control of emotional experience through control of expressions [ J ] . Cognition \& Emotion, 2001, 15 ( 1 ) : 27-56. https://doi.org/10.1080/02699930126057

[11 ] Bush L K, Barr C L, Mchugo G J, et al. The Effects of Facial Control and Facial Mimicry on Subjective Reactions to Comedy Routines [ J ] . Motivation and Emotion, 1989, 13 ( 1 ) : 31-52. https://doi.org/10.1007/BF00995543

[12 ] Mccanne T R, Anderson J A. Emotional responding following experimental manipulation of facial electromyographic activity. [ J ] . Journal of Personality and Social Psychology, 1987, 52 ( 4 ) : 759-768.

https://doi.org/10.1037//0022-3514.52.4.759

[13] Levenson R W, Ekman P, Friesen W V. Voluntary Facial Action Generates Emotion-Specific Autonomic Nervous System Activity [ J ] . Psychophysiology, 1990, 27 ( 4 ) : 363-384. https://doi.org/10.1111/j.1469-8986.1990.tb02330.x

[14 ] Izard C E. The face of emotion [ J ] . Suny, 1971 ( 1 ) : 112.

[ 15 ] Riskind J H, Gotay C C. Physical Posture: Could It Have Regulatory or Feedback Effects on Motivation and Emotion? [ J ] . Motivation and Emotion, 1982, 6 ( 3 ) : 273-298. https://doi.org/10.1007/BF00992249

[16] Wallington S A. Consequences of transgression self-punishment and depression [ J ] . Journal of Personality \& Social Psychology, 1973, 28 ( 1 ) : 1. https://doi.org/10.1037/h0035576

[ 17 ] Riskind J H. They stoop to conquer: Guiding and self-regulatory functions of physical posture after success and failure $[\mathrm{J}]$. Journal of Personality and Social 
Psychology, 1984, 47 ( 3 ) : 479-493.

https://doi.org/10.1037//0022-3514.47.3.479

[18 ] Michalak J, Mischnat J, Teismann T. Sitting posture makes a differenceembodiment effects on depressive memory bias [ J ] . Clinical Psychology \& Psychotherapy, 2014, 21 (6) : 519-524. https://doi.org/10.1002/cpp.1890

[19] Veenstra L, Schneider I K, Koole S L. Embodied mood regulation: the impact of body posture on mood recovery, negative thoughts, and moodcongruent recall $[\mathrm{J}]$. Cognition and Emotion, 2016: 1-16. https://doi.org/10.1080/02699931.2016.1225003

[20 ] Nair S, Sagar M, Sollers J, et al. Do slumped and upright postures affect stress responses? A randomized trial. [ J ] . Health Psychology, 2015, 34 ( 6 ) : 632-641. https://doi.org/10.1037/hea0000146

[21] Shafir T, Taylor S F, Atkinson A P, et al. Emotion regulation through execution, observation, and imagery of emotional movements $[\mathrm{J}]$. Brain and Cognition, 2013, 82( 2 ): 219-227. https://doi.org/10.1016/j.bandc.2013.03.001

[22 ] Koole S L, Veenstra L. Does Emotion Regulation Occur Only Inside Peoplel"s Heads? Toward a Situated Cognition Analysis of Emotion-Regulatory Dynamics [ J ] . Psychological Inquiry, 2015, 26 ( 1 ) : 61-68. https://doi.org/10.1080/1047840X.2015.964657

[23 ] Kamila O, Evan T, Wayne P M. Can Tai Chi and Qigong Postures Shape Our Mood? Toward an Embodied Cognition Framework for Mind-Body Research [ J ] . Frontiers in Human Neuroscience, 2018, 12: 174-186.

https://doi.org/10.3389/fnhum.2018.00174

[24] Miragall M, Etchemendy E, Ausi à s Cebolla, et al. Expand your body when you look at yourself: The role of the posture in a mirror exposure task $[\mathrm{J}]$. Plos One, 2018, 13 ( 3 ) . https://doi.org/10.1371/journal.pone.0194686

[25] Eriksson S, Gard G. Physical exercise and depression [ J ]. Physical Therapy Reviews, 2013, 16 ( 4 ) : 261-268. 


\section{https://doi.org/10.1179/1743288X11Y.0000000026}

[26] Wilkes C, Kydd R, Sagar M, et al. Upright posture improves affect and fatigue in people with depressive symptoms $[\mathrm{J}]$. Journal of Behavior Therapy and Experimental Psychiatry, 2017, 54: 143-149.

https://doi.org/10.1016/j.jbtep.2016.07.015

[27 ] Winkielman P, Niedenthal P, Wielgosz J, et al. Embodiment of cognition and emotion[ M ]//APA handbook of personality and social psychology, Volume 1: Attitudes and social cognition. Washington, DC: American Psychological Association, 2015. https://doi.org/10.1037/14341-004

[28 ] Dijkstra K, Kaschak M P, Zwaan R A. Body posture facilitates retrieval of autobiographical memories [ J ] . Cognition, 2007, 102 ( 1 ) : 139-149. https://doi.org/10.1016/j.cognition.2005.12.009

[ 29 ] Coyne J C, Gotlib I H. The role of cognition in depression: A critical appraisal[ J ]. Psychological Bulletin, 1983, 94 ( 3 ) : 472-505.

https://doi.org/10.1037//0033-2909.94.3.472 